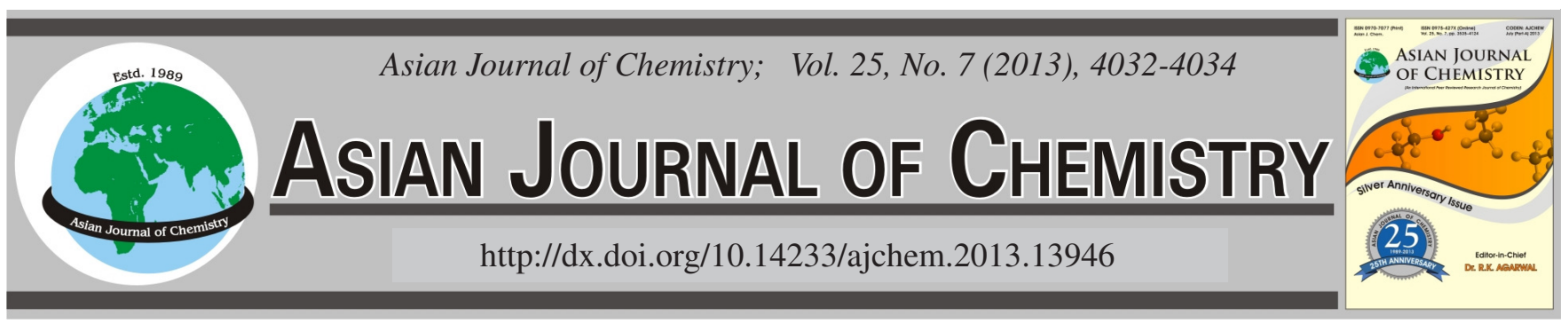

\title{
Preparation of Poly(methyl methacrylate) Functionalized Carbon Fiber via Reversible Addition-Fragmentation Chain Transfer Polymerization
}

\author{
Lei Xiong ${ }^{*}$, Xiaofeng Li, Hongbo Liang* and Shengmei Huang
}

School of Material Science and Engineering, Nanchang Hangkong University, Nanchang 330063, P.R. China

*Corresponding author: Tel: +86 791 83863025; E-mail: x_lei81@163.com; liahongbo@163.com

\begin{abstract}
We report a novel method for the functionalization of carbon fiber with poly(methyl methacrylate) by using reversible addition-fragmentation chain transfer polymerization. The carbon fiber was first oxidized with a mixture of nitric acid and sulphuric acid (1:3), in order to introduce hydroxyl groups onto the carbon fiber surface. Then the reversible addition-fragmentation chain transfer agents were immobilized onto the surface of carbon fiber by chemical reaction of hydroxyl groups with silane coupling agent containing dithioester groups. Finally, the methyl methacrylate was initiated and propagated on the carbon fiber surface by reversible addition-fragmentation chain transfer. The poly(methyl methacrylate) grafted carbon fiber was characterized by FT-IR, ${ }^{1} \mathrm{H}$ NMR and TGA. The results show that the poly(methyl methacrylate) grafted carbon fiber were synthesized successfully and the graft content could reach $32.4 \%$.
\end{abstract}

Key Words: Carbon fiber, Reversible addition-fragmentation chain transfer, Poly(methyl methacrylate), Surface modification.

ᄂ - - - - - - - - - - - - - - - - - - - - - - - - - - -

\section{INTRODUCTION}

In the past decade, carbon fibers (CF) have attracted extensively attention because of their high specific strength, high stiffness and chemical stability, which have been used in many fields, such as aerospace industry, automotive industry, steamship industry, petroleum and chemical industry ${ }^{1,2}$. Unfortunately, they have difficulty in forming a strong adhesive bond with polymer matrix due to their smooth surface and chemical inert nature ${ }^{3-5}$. Nowadays, since carbon fibers reinforced polymer composites become more and more important, several surface treatments are developed to modify carbon fibers surface, including chemical oxidation, plasma activation, electrochemical treatments and surface functional groups grafting procedure ${ }^{6-11}$. Among these methods, grafting polymer on carbon fibers is an effective way to provide a strong interfacial bond between carbon fiber surface and polymer.

In this paper, we focused on the study about the modification of carbon fiber with poly(methyl methacrylate) (PMMA) via reversible addition-fragmentation chain transfer polymerization (RAFT) for the purpose of improving the fibermatrix interfacial adhesion. The functionalized carbon fibers with PMMA were studied by means of FT-IR, ${ }^{1} \mathrm{H}$ NMR and TGA.

\section{EXPERIMENTAL}

Carbon fibers used in this study were obtained from Hexcel. Methyl methacrylate (MMA) was purchased from
Shanghai Reagents Co., Ltd. and was distilled under reduced pressure to remove the inhibitor before polymerization. 2,2'Azobisisobutyronitrile (AIBN) obtained from Aldrich was recrystallized with ethanol before use. 3-(Chloropropyl) triethoxy silane and the sodium ethyl xanthate were purchased from Arcos and used to synthesis silane coupling agent containing dithioester groups.

Synthesis of carbon fibres-reversible addition-fragmentation chain transfer agent: For the chemical oxidation of carbon fiber, the purified carbon fiber was mixed with the solution of conc. nitric acid and sulphuric acid in 1:3 ratio and the mixture was ultra sonicated for $8 \mathrm{~h}$. The resulted fiber was then diluted with a slow and continuous flow of distilled water for $10 \mathrm{~h}$, centrifuged at $4000 \mathrm{rpm}$ and washed with deionized water. The oxidized carbon fibers were then modified by silane coupling agent with RAFT agent. At the end of reaction, the product was vacuum filtered through a $0.22 \mu \mathrm{m}$ poly(tetrafluoroethylene) (PTFE) membrane and the functionalized carbon fiber was washed with anhydrous ethanol for three times. The RAFT agent functionalized carbon fiber [CF-SC(S)OC ${ }_{2} \mathrm{H}_{5}$ ] obtained was dried under vacuum at $45^{\circ} \mathrm{C}$ for $36 \mathrm{~h}$.

Surface modification of carbon fibers by poly(methyl methacrylate via reversible addition-fragmentation chain transfer: For a typical experiment, a mixture of CF-SC(S)OC $\mathrm{OH}_{2}$, MMA, AIBN and DMF was added into a dried flask. The content was purged with argon for $10 \mathrm{~min}$ to eliminate the oxygen and then the flask was sealed. The polymerization was performed 
under ultrasonic irradiation. After a prescribed reaction time, the flask was cooled with ice water and opened. The reaction mixture was diluted with tetrahydrofuran (THF) and vacuum filtered with $0.22 \mu \mathrm{m}$ PTFE membrane. The resulting filtered product was redispersed in $10 \mathrm{~mL}$ of THF, precipitated by the addition of abundant methanol and dried under vacuum at room temperature for $24 \mathrm{~h}$. The procedure of surface modification of carbon fibers by PMMA is presented in Fig. 1.
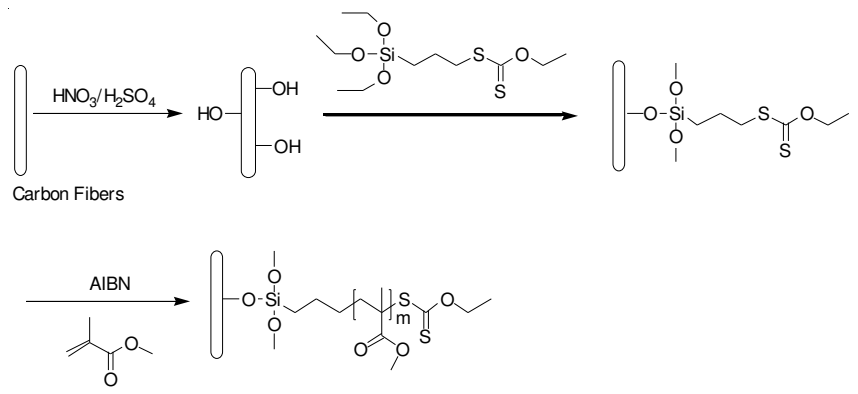

Fig. 1. Surface modification of carbon fibers

${ }^{1} \mathrm{H}$ Nuclear magnetic resonance (NMR) spectrum was recorded on a Bruker DMX 500 spectrometer in $\mathrm{CDCl}_{3}$. Fourier transform infrared (FT-IR) spectra of the products were conducted on a Bruker 210 IR spectrometer at room temperature. Thermal gravimetric analysis (TGA) was recorded on a Shimadzu TG-50. The samples were heated from $25-700{ }^{\circ} \mathrm{C}$ at a heating rate of $20^{\circ} \mathrm{C} / \mathrm{min}$ under nitrogen atmosphere.

\section{RESULTS AND DISCUSSION}

Reversible addition-fragmentation chain transfer agents grafting on the carbon fiber: The covalent bond of RAFT agents on the surface was determined through FT-IR spectroscopy. Fig. 2 presents the FT-IR spectra of pristine carbon fiber and $\mathrm{CF}-\mathrm{SC}(\mathrm{S}) \mathrm{OC}_{2} \mathrm{H}_{5}$. Compared with the pristine carbon fiber, $\mathrm{CF}-\mathrm{SC}(\mathrm{S}) \mathrm{OC}_{2} \mathrm{H}_{5}$ spectrum shows a broad absorption peak at $3500-3100 \mathrm{~cm}^{-1}$ attributed to vibrations of the -OH groups on the carbon fiber surface. The broad absorption peaks at 2950 and $2865 \mathrm{~cm}^{-1}$ are due to the aliphatic hydrogen of the grafted RAFT agents. The two well resolved peaks which appeared at 1220 and $1050 \mathrm{~cm}^{-1}$ are assigned to the stretching vibrations of $\mathrm{C}-\mathrm{O}$ and $\mathrm{C}=\mathrm{S}$, respectively. From the FT-IR results, it can be deduced that RAFT agents have been grafted on the carbon fiber surface through the formation of covalent bonds. The dithioester groups of the RAFT agents can further react with MMA via the RAFT process to form a polymer chemically bonded to the carbon fiber surface.

Grafting PMMA from the carbon fibers surface: The carbon fibers functionalized with PMMA (CF-PMMA) were prepared by RAFT process. For the FT-IR spectrum of CFPMMA in the Fig. 3, peak at $1730 \mathrm{~cm}^{-1}$ which is attributable to $\mathrm{C}=\mathrm{O}$ stretching vibrations of ester units appears. The peak at $2950 \mathrm{~cm}^{-1}$ is due to the $\mathrm{C}-\mathrm{H}$ bending. In addition to, the peak at $1150 \mathrm{~cm}^{-1}$ can be assigned to the $\mathrm{C}-\mathrm{O}$ stretching of the ester group. The FT-IR data confirm the presence of PMMA chains on the carbon fiber surface.

The covalent bonding of PMMA onto the carbon fiber surface is further supported by NMR. The ${ }^{1} \mathrm{H}$ NMR spectrum

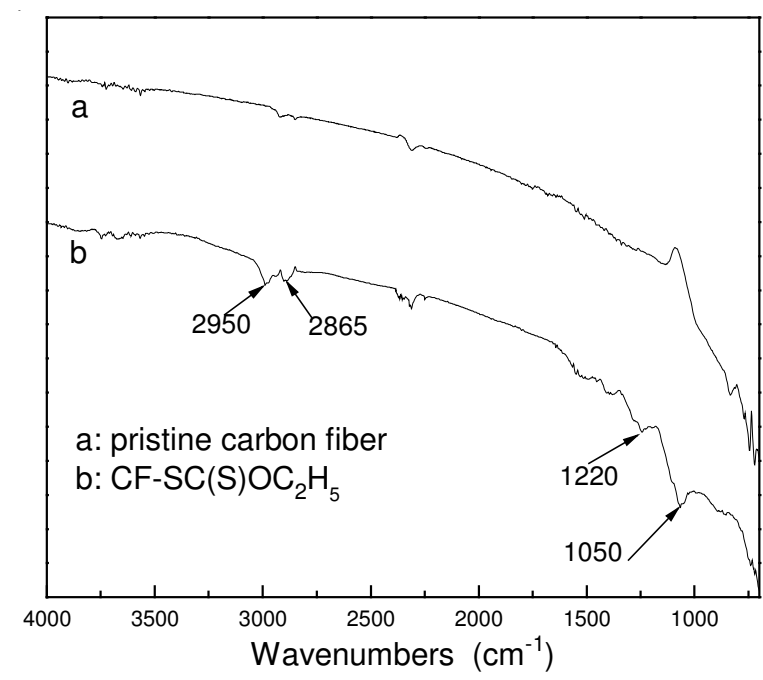

Fig. 2. FT-IR spectra of pristine carbon fiber and CF- SC(S)OC $\mathrm{O}_{2} \mathrm{H}_{5}$

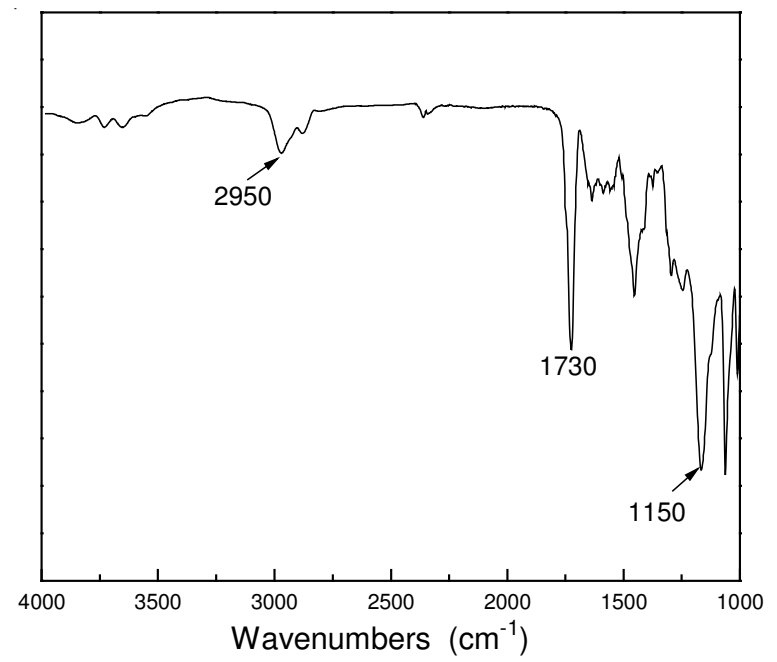

Fig. 3. FT-IR spectrum of CF-PMMA

of PMMA cleaved from the carbon fiber surface is shown in the Fig. 4. The signals at 0.7-1 ppm are assigned to the protons of methyl groups adjacent to the backbone. The signal at 3.4 ppm is assigned to methyl protons of methyl ester group. The peaks at $1.8 \mathrm{ppm}$ are also observed. We assign these peaks to the proton of the methylene groups in the backbone of the PMMA chains. In addition, the signal at $2.6 \mathrm{ppm}$ is attributable to the methylene protons of the grafted RAFT agent.

The amount of grafted polymer on the carbon fiber surface was determined through the TGA analysis by comparing the TGA traces of pristine carbon fiber, $\mathrm{CF}-\mathrm{SC}(\mathrm{S}) \mathrm{OC}_{2} \mathrm{H}_{5}$ and CFPMMA and the results are showed in Fig. 5. The sample of pristine carbon fiber decomposes slowly with the increasing of temperature because of the losing of functional groups or absorbed water on the carbon fiber surface and the weight loss is about $5.4 \%$ between 25 and $700{ }^{\circ} \mathrm{C}$. On the contrary, the sample of CF-SC(S)OC $\mathrm{OH}_{5}$ displays weight loss of about $13.2 \%$. This weight loss results from the losing of RAFT agent on the surface of carbon fiber and the weight loss is consistent with the content of RAFT agent grafted onto the carbon fiber. Compared with the pristine carbon fiber and CF-SC(S)OC ${ }_{2} \mathrm{H}_{5}$, the TGA curve of CF-PMMA showed three main weight loss 


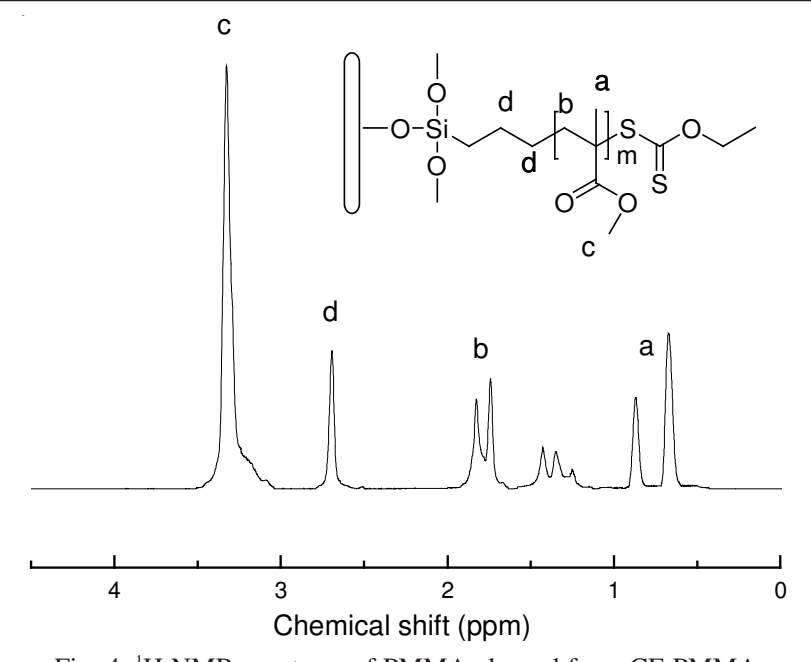

Fig. 4. ${ }^{1} \mathrm{H}$ NMR spectrum of PMMA cleaved from CF-PMMA

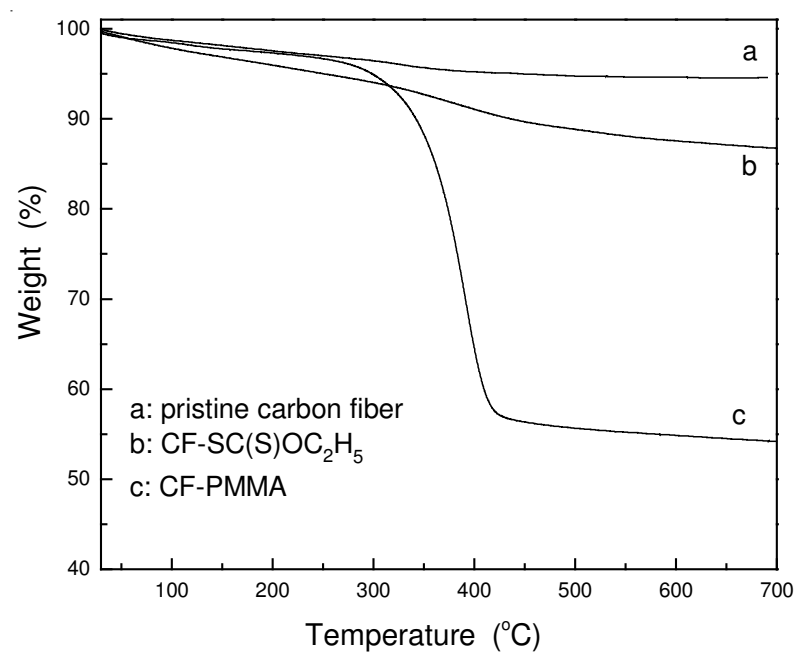

Fig. 5. TGA curves of different carbon fibers

regions. The first weight loss region below $250{ }^{\circ} \mathrm{C}$ can be attributed to the decomposition of the functional groups on the carbon fiber surface. The second region $\left(250-400^{\circ} \mathrm{C}\right)$ shows a rapid weight reduction which may come from the decomposition of PMMA and RAFT agent. A little loss in weight in the third region $\left(400-700{ }^{\circ} \mathrm{C}\right)$ can be assigned to the further decomposition of polymer segments. The amount of polymer calculated from TGA data is $c a .32 .4 \%$.

\section{Conclusion}

In this study, the preparation of RAFT agent grafted onto the carbon fiber surface and the functionalization of carbon fiber with PMMA via RAFT polymerization were studied. After the RAFT agents were introduced onto the carbon fiber surface, the PMMA chains were successfully grafted from the carbon fiber surface via RAFT polymerization. The resulted products were characterized by means of FT-IR, ${ }^{1} \mathrm{H}$ NMR and TGA. The results demonstrated that the PMMA was successfully grafted from the carbon fiber surface and the amount of the polymer grafted on the carbon fiber is $c a .32 .4 \%$. The realization of polymerization of MMA monomers on the carbon fiber surface by the convenient RAFT "grafting from" strategy would pave the way for the fabrication and application of more carbon fiber-polymer composites.

\section{ACKNOWLEDGEMENTS}

The authors gratefully acknowledged the financial support of the National Natural Science Foundation of China (No. 51103069), Foundation of Jiangxi Educational Committe (No. GJJ12423), Foundation of Aeronautics of China (No. 2011 ZF56018) and Natural Science Foundation of Jiangxi Province (No. 20122BAB216014).

\section{REFERENCES}

1. Z.R. Yue, W. Jiang, L. Wang, S.D. Gardner and C.U. Pittman Jr., Carbon, 37, 1785 (1999).

2. C. Marieta, E. Schulz, L. Irusta, N. Gabilondo, A. Tercjak and I. Mondragon, Comp. Sci. Technol., 65, 2189 (2005).

3. S. Yumitori and Y. Nakanishi, Composites, 27, 1059 (1996).

4. F. Severini, L. Formaro, M. Pegoraro and L. Posca, Carbon, 40, 735 (2002).

5. M.H. Choi, B.H. Jeon and I.J. Chung, Polymer, 41, 3243 (2000).

6. M.D. Garcia, F.J. Lopez-Garzon and M. Perez-Mendoza, J. Colloid. Interf. Sci., 222, 233 (2000).

7. H.L. Cao, Y.D. Huang and Z.Q. Zhang, Comp. Sci. Technol., 65, 1655 (2005).

8. C.U. Pittman Jr., G.R. He, B. Wu and S.D. Gardner, Carbon, 35, 317 (1997).

9. M.B. Ivanov, N.V. Gavrilov, T.A. Belyh, E.A. Ligacheva, L.V. Galijeva, A.E. Ligachev and V.V. Sohoreva, Surf. Coat. Technol., 201, 8326 (2007).

10. N. Dilsiz, N.K. Erinc, E. Bayramli and G. Akovali, Carbon, 33, 853 (1995).

11. A.S. Sarac and Y. Bardavit, Prog. Org. Coat., 49, 85 (2004). 